\title{
HIIT or miss: Is high-intensity interval training (HIIT) the way forward for obese children?
}

Danielle Lambrick, $\mathrm{PhD}^{1}$; Lee Stoner, PhD MPH FRSPH ${ }^{2}$; James Faulkner, $\mathrm{PhD}^{3}$

\footnotetext{
${ }^{1}$ Faculty of Health Sciences, University of Southampton, United Kingdom

${ }^{2}$ School of Sport and Exercise, Massey University, Wellington, New Zealand.

${ }^{3}$ Department of Sport and Exercise, University of Winchester, United Kingdom
}
* Corresponding Author:
E: D.M.Lambrick@soton.ac.uk
T: +44 (0)2380595916
F: $+44(0) 2380593131$

Type of article: opinion piece on topical issues in public health

Conflicts of interest: NONE

Source of funding: The author(s) received no financial support for the research, authorship, and/or publication of this article.

Contributions: All authors aided with the writing and critically revised the manuscript for important intellectual content. DL is the guarantor. All authors gave final approval for publication. 


\section{INTRODUCTION}

Globally, there is a continual increase in the prevalence of childhood obesity, ${ }^{1}$ placing children at increased risk of premature onset of non-communicable diseases (NCDs), including musculoskeletal disorders, some cancers, and cardio-metabolic diseases such as type 2 diabetes and cardiovascular diseases (CVD). ${ }^{1}$ This rise in obesity and co-morbid complications has been strongly linked to lifestyle choices, including physical inactivity ${ }^{2}$. However, despite heightened public awareness of the relationship between physical inactivity and obesity, rates of physical activity are not improving. ${ }^{3}$ An often cited barrier to physical activity among both adults and children is a lack of time, ${ }^{4}$ which has led to the promotion of high-intensity interval training (HIIT) as a more time-efficient exercise prescription strategy.

A recent meta-analysis by García-Hermoso and colleagues ${ }^{4}$ has examined the limited number of studies investigating the efficacy of HIIT in overweight and obese youth. HIIT was not reported to be superior to other forms of exercise when examining changes in body composition, but HIIT was suggested to be more effective for improving systolic blood pressure (SBP) and cardio-respiratory fitness. These findings indicate that HIIT may be effective for improving cardio-metabolic health and thus, decreasing the risk of NCDs. However, these physiological findings need to be considered in the context of clinical inference, implementation science, and the potential for public health outcomes.

\section{CLINICAL INFERENCE}

Prior to discussing the implementation of HIIT, the potential clinical implications should be contextualized. As a primary outcome, the meta-analysis by García-Hermoso and colleagues ${ }^{4}$ utilised the gold-standard measure of cardio-respiratory fitness: maximal oxygen uptake $\left(\dot{\mathrm{V}}_{2} \mathrm{max}\right)$. When compared to other forms of exercise, $\dot{\mathrm{V}} \mathrm{O}_{2}$ max improved, on average, by $1.92 \mathrm{ml} \cdot \mathrm{kg}^{-1} \cdot \mathrm{min}^{-1}$. While findings in children are inconclusive, ${ }^{5,6}$ cardiorespiratory fitness has been recognized as a more important correlate of cardio-metabolic health than obesity in adults. ${ }^{7}$ However, $1.92 \mathrm{ml} \cdot \mathrm{kg}^{-1} \cdot \mathrm{min}^{-1}$ is greater than the measurement error for $\mathrm{V}_{2}$ max testing and it is thus unlikely that such a small change is clinically meaningful in terms of risk stratification.

García-Hermoso and colleagues ${ }^{4}$ also reported that SBP improved, on average, by $3.67 \mathrm{mmHg}$. High SBP (hypertension) is associated with earlier onset of cardiovascular diseases ${ }^{8}$ and, according to the World Health 
Organization (WHO), is likely the leading risk factor for death worldwide. ${ }^{9}$ While child-specific data is unavailable, in adults a $20 \mathrm{mmHg}$ increase in SBP is associated with a two-fold increase in risk of death from coronary artery disease or stroke. ${ }^{10}$ However, a value of $3.67 \mathrm{mmHg}$ is greater than the measurement error of most blood pressure devices, ${ }^{11}$ and it is difficult to discern the clinical importance of such a small change. The authors do also report on several markers of body composition, blood lipids and insulin resistance; none of which were found to be statistically different or clinically meaningful. Nonetheless, HIIT has been reported to result in clinically meaningful improvements in cardiovascular health among adults, ${ }^{12}$ so this form of exercise prescription does hold potential, and discussion is warranted in terms of implementation and public health outcomes.

\section{IMPLEMENTATION AND PUBIC HEALTH}

While HIIT prescription may overcome the time barrier to exercise adherence, prior to advocating HIIT at a public health level, there are a number of important questions regarding its utility that remain unanswered, including, but not limited to: is this form of exercise really appropriate for obese children? In particular, will this form of exercise prescription put obese children at increased risk for musculoskeletal injuries? Should some form of priming period precede the implementation of HIIT, i.e., should HIIT be employed following a period of strength training; which has been shown to improve cardio-respiratory fitness and psychological wellness in obese children, and is well-tolerated ${ }^{13,14}$ Considering children undergo major gender-specific physiological changes when transitioning to adolescence, ${ }^{2}$ should HIIT be tailored to the age and gender of the child? While limited evidence in older men indicates that HIIT training may improve motivation to exercise, ${ }^{15}$ is this form of exercise enjoyable and sustainable for children? Lastly, what kinds of physical resources and practitioner training are required to implement HIIT at the community level, and should school physical educators receive such training?

\section{CONCLUSIONS}

While there is physiological evidence to support the use of HIIT in some adult populations, the child-based evidence is inconclusive. Further, there is scant discussion with regards to implementation and public health outcomes, particularly for overweight and obese children. As such, there is currently insufficient physiological 
or practical evidence to recommend an update to childhood physical activity guidelines. Prior to advocating HIIT for overweight and obese children, there are a number of questions that need to be addressed in order to determine the physiological and psychosocial benefits of such exercise, as well the feasibility of its implementation. Thus, although not a miss, we are still a way off HIIT being a true hit! 


\section{REFERENCES}

1. WHO. Plan of action of the Commission on Ending Childhood Obesity. World Health Organization, 2014.

2. Wu BW, Skidmore PM, Orta OR, et al. Genotype vs. Phenotype and the Rise of Non-Communicable Diseases: The Importance of Lifestyle Behaviors During Childhood. Cureus. 2016; 8: e458.

3. Hallal PC, Andersen LB, Bull FC, Guthold R, Haskell W and Ekelund U. Global physical activity levels: surveillance progress, pitfalls, and prospects. Lancet. 2012; 380: 247-57.

4. Garcia-Hermoso A, Cerrillo-Urbina AJ, Herrera-Valenzuela T, Cristi-Montero C, Saavedra JM and Martinez-Vizcaino $\mathrm{V}$. Is high-intensity interval training more effective on improving cardiometabolic risk and aerobic capacity than other forms of exercise in overweight and obese youth? A metaanalysis. Obes Rev. 2016.

5. Moschonis G, Mougios V, Papandreou C, et al. "Leaner and less fit" children have a better cardiometabolic profile than their "heavier and more fit" peers: The Healthy Growth Study. Nutr Metab Cardiovasc Dis. 2013; 23: 1058-65.

6. Stoner L, Lambrick DM and Faulkner J. We're not ready to encourage children to be "lean" rather than "fit". Nutr Metab Cardiovasc Dis. 2014; 24: e6-7.

7. Hainer V, Toplak H and Stich V. Fat or fit: what is more important? Diabetes Care. 2009; 32 Suppl 2: S392-7.

8. Franco $\mathrm{OH}$, Peeters $\mathrm{A}$, Bonneux $\mathrm{L}$ and de Laet $\mathrm{C}$. Blood pressure in adulthood and life expectancy with cardiovascular disease in men and women: life course analysis. Hypertension. 2005; 46: 280-6.

9. WHO. The World Health Report 2002. Geneva: World Health Organization (WHO), 2002.

10. Chobanian AV, Bakris GL, Black HR, et al. Seventh report of the Joint National Committee on Prevention, Detection, Evaluation, and Treatment of High Blood Pressure. Hypertension. 2003; 42: 1206-52.

11. Young $\mathrm{Y}, \mathrm{Abdolhosseini} \mathrm{P}, \mathrm{Brown} \mathrm{F}$, et al. Reliability of oscillometric central blood pressure and wave reflection readings: effects of posture and fasting. J Hypertens. 2015; 33: 1588-93.

12. Ramos JS, Dalleck LC, Tjonna AE, Beetham KS and Coombes JS. The impact of high-intensity interval training versus moderate-intensity continuous training on vascular function: a systematic review and meta-analysis. Sports Med. 2015; 45: 679-92.

13. LeMura LM and Maziekas MT. Factors that alter body fat, body mass, and fat-free mass in pediatric obesity. Med Sci Sports Exerc. 2002; 34: 487-96.

14. Shultz SP, Stoner L, Lambrick DM and Lane AM. A boxing-oriented exercise intervention for obese adolescent males: findings from a pilot study. Journal of sports science \& medicine. 2014; 13: 751-7.

15. Knowles AM, Herbert P, Easton C, Sculthorpe N and Grace FM. Impact of low-volume, high-intensity interval training on maximal aerobic capacity, health-related quality of life and motivation to exercise in ageing men. Age (Dordr). 2015; 37: 25. 